\title{
Las emociones de la mujer como estrategia publicitaria del campo farmacéutico a comienzos del siglo XX en Chile*
}

\author{
Mario Millones Espinosa* \\ Nicolás Hernández Maluenda*** \\ Recibido: 2019-06-15 • Enviado a pares: 2019-06-28 \\ Aprobado por pares: 2019-07-28 • Aceptado: 2019-09-20 \\ https://doi.org/10.22395/angr.v18n36a5
}

\begin{abstract}
Resumen
El presente artículo tiene como objetivo reflexionar sobre cómo las emociones formaron parte de la expansión del campo farmacéutico a comienzos del siglo xx en Chile. Para introducir el consumo de medicamentos en el hogar, distintas boticas y farmacias publicitaron sus remedios en revistas como Familia. Allí se dieron dos hechos importantes: primero, el uso paulatino de las emociones en los anuncios publicitarios de los fármacos y, segundo, el recurrir a las mujeres de forma constante en esta estrategia. A través de ella en tanto mujer, madre y sus distintas obligaciones morales inscritas en tales roles, creemos que la medicalización de la familia necesitó de cierto argumento emocional para la expansión del campo farmacéutico.
\end{abstract}

Palabras clave: medicalización; publicidad; medicamentos; salud; emociones.

El presente artículo es el resultado de la investigación auxiliar de la tesis doctoral en Sociología de Mario Millones Espinosa en la Universidad Alberto Hurtado, Santiago de Chile, financiada por Conicyt y el Gobierno de Chile.

** Doctor en Sociología, Universidad Alberto Hurtado, Santiago, Chile. Correo electrónico: mario.millones.espinosa@gmail.com

".. Sociólogo, Universidad de Valparaíso, Chile. Investigador asistente en SurAndes Consultores, Chile. Correo electrónico: nicolas.hernandezm12@gmail.com 


\title{
Woman's Emotions as an Advertising Strategy of the Pharmaceutical Field at the Beginning of the $20^{\text {th }}$ Century in Chile
}

\begin{abstract}
This article aims to reflect on how emotions were part of the expansion of the pharmaceutical field in the early twentieth century in Chile. To introduce the consumption of medicines at home, different pharmacies and apothecaries advertised their remedies in magazines like Family. Two important events took place: first, the gradual use of emotions in drug advertisements and second, the constant use of women in this strategy. Through her, as a woman and mother, and the different moral obligations inscribed in such roles, that we believe that the medicalization of the family needed some emotional argument for the expansion of the pharmaceutical field.
\end{abstract}

Keywords: medicalization; advertising; medicine; health; emotion.

\section{As emoções da mulher como estratégia publicitária do campo farmacêutico no início do século XX no Chile}

\begin{abstract}
Resumo
Este artigo tem como objetivo refletir sobre como as emoções fizeram parte da expansão do campo farmacêutico no início do século xx, no Chile. Para introduzir o consumo de medicamentos nos lares, diferentes boticas e farmácias fizeram publicidade de seus remédios em revistas como a Familia. Nela, aconteceram dois fatos importantes: primeiro, o uso paulatino das emoções nos anúncios publicitários dos farmacêuticos; segundo, o recurso às mulheres de forma constante nessa estratégia. Com base na mulher e na mãe, e em suas diferentes obrigações morais vinculadas a esses papéis, acreditamos que a medicalização da família precisou de certo argumento emocional para a expansão do campo farmacêutico.

Palavras-chave: medicalização; publicidade; medicamentos; saúde; emoções.
\end{abstract}




\section{Introducción}

A comienzos del siglo xx apareció en Chile la revista Familia, un magazín ${ }^{1}$ de publicación mensual dedicado al entretenimiento de las mujeres pertenecientes a hogares de clase alta. Allí, la idea de mujer moderna se transformó en el articulador de una línea editorial que comenzaba a ser popular en Estados Unidos y Europa principalmente (Schwartz, 2011). Familia apareció en 1910 y se publicó hasta 1928. Desapareció varios años y volvió a surgir en 1935 hasta 1940, año en que terminó su impresión. Perteneció a la editorial Zig-Zag del empresario Agustín Edward, dueño también del periódico El Mercurio.

Desde un comienzo, Familia se dedicó al hogar, la mujer y la familia: cómo debía vivir la mujer dentro y fuera del hogar, cómo debía lucir su cuerpo, sus atuendos y peinados importados desde Europa; qué gustos debía tener para decorar la casa, qué comidas preparar para ciertas ocasiones y cómo cuidar a sus hijos. Muchos de estos aspectos parecen bien trabajados por Orellana (2016) en cuanto a aquellos artilugios para la configuración estética y social de la mujer de comienzos de siglos, adicionalmente, en algunos casos aparecía también información médico-farmacéutica como fundamento.

Ahora bien, la idea de entregar consejos para la vida no fue propiedad exclusiva de la revista Familia. Situación similar presentaron otras revistas como 18 (Alliende, 1987) destinada según Sánchez (2016), como manual de instrucciones prácticas para la vida bajo la mirada farmacéutica. Se podría señalar algo similar con Hoja Sanitaria, un pasquín anarquista que bajo argumentos médicos intentó educar a los trabajadores y sus familias en distintos temas y problemas relacionados con la salud (Fuster y Moscoso-Flores, 2015), entre otras revistas.

No obstante, nos enfocaremos solo en la revista Familia debido al rol que cumple la mujer en esta. Si bien en los otros también aparecía la mujer, en Familia, constituyó un personaje central en cada una de sus ediciones debido a su línea editorial y ello nos interesa como línea de investigación en la medicalización de la vida cotidiana. Allí, un aspecto que se comienza a perfilar es el uso de las emociones como estrategia de publicidad que utiliza el campo farmacéutico para legitimar sus productos.

En diálogo entre lo que Illouz (2007) denomina como capitalismo emocional con el concepto de Anderson (1991) del capitalismo impreso, creemos que el formato magazín fue un agente clave para la legitimación de las tecnologías farmacéuticas -entre otras-y su proceso de domesticación. En este artículo analizaremos algunos

\footnotetext{
El magazín corresponde a un modo específico de relación cultural y pública de masas populares (Ossandón y Santa Cruz, 2005) de finales del siglo xix y primeras décadas del xx, en las que además de informar se señalaban aspectos sobre cómo se debía vivir. Familia, por su lado, emergió en un periodo que Montero (2013) señala como el primero de la historia de la prensa de mujeres en Chile (García Huidobro y Escobar, 2012; Orellana, 2016), por lo que su relevancia en este sentido no será menor.
} 
anuncios publicitarios que aparecen en la revista Familia en la que se aprecia el uso de emociones como estrategia del campo farmacéutico para expandir su dominio sobre la vida cotidiana.

La selección de anuncios publicitarios que aquí se muestran no representa una muestra aleatoria probabilística sino intencionada bajo el criterio de que los anuncios muestren de forma explícita la idea que desarrollamos. La aparición de la mujer y el apelar a las emociones como estrategia fue un movimiento paulatino que ayudó, entre otros, a la farmaceuticalización de la vida (Abraham, 2010). Pretender señalar que ello fue la gran y única estrategia o razón para el consumo de medicamentos sería reducir la complejidad de un fenómeno amplio y lleno de aristas como la medicalización de la vida en general (Conrad, 2007). Por ello, este artículo constituye una reflexión ensayística del devenir en la relación medicamentos y emociones².

Así, creemos que el proceso de "domesticación de fármacos" (Fox y Ward, 2008) tuvo una transición en la que las emociones fueron ocupando un espacio esencial para este mercado en el que los testimonios de personas que gozan sus efectos y consejos de médicos como garantía de estos (Correa, 2016) legitiman el fármaco.

Para mostrar lo anterior, hacemos primero hincapié en la emergencia del campo farmacéutico en Chile. Luego, entramos a conocer la revolución tecnológica del hogar y su impacto en las emociones en las mujeres-madres para, posteriormente, hacer énfasis en el giro de la publicidad hacia el mundo emocional de las mujeres a comienzos del siglo XX en Chile. Por último, analizamos la relación entre capitalismo y emociones bajo este panorama de la domesticación de los medicamentos.

\section{La emergencia del campo farmacéutico en Chile}

En los inicios del oficio, los primeros boticarios tuvieron una fuerte influencia, tanto de migrantes que trajeron sus propios conocimientos específicos (Meyer, 1986), como del saber medicinal de culturas indígenas locales que usaron una gran cantidad de plantas autóctonas con fines terapéuticos. En tiempos de la Colonia, la botica de los jesuitas era el gran espacio de creación, divulgación y venta de remedios elaborados localmente o importados (Guzmán, 2003).

Con la instalación de la República, la farmacéutica adquirió un nuevo aire. Se comenzaron a producir grandes obras de naturalistas que recorrían el país estudiando la flora nacional con la idea de conocer sus distintas propiedades para identificar cuáles podían ser beneficiosas para la población en general. Por ejemplo, en 1845 se publicó Botánica, compuesta por ocho tomos. Allí, la cantidad de especies descritas para el país alcanzó "3.767 de todos los grupos" (Jiménez et al., 2016, p. 37). Años después

Relación que uno de los autores, Mario Millones Espinosa, explora en su tesis doctoral en Sociología de la Universidad Alberto Hurtado en relación con el consumo de medicamentos dentro del mundo laboral. 
y de forma complementaria, en 1869 Rodulfo Philippi escribió Elementos de Botánica (Jiménez et al., 2016, p. 37). En 1889 el médico Adolfo Murillo publicó un catálogo llamado Plantes medicinales du Chile, en el que se concentró solo en aquellas plantas que fueran originarias de Chile (Jiménez et al., 2016, p. 37).

Adolfo Murillo y Carlos Middleton publicaron en 1886 la obra Famacopea chilena como un intento de uniformar todos estos contenidos. El objetivo de esta nueva obra era describir aquellos fármacos que se usaban cotidianamente y sacar del listado los que ya se hallaban en desuso para la época. La obra fue oficializada y "arrojó que de un total de 897 preparados, 577 correspondían a plantas, de las cuales 59 eran plantas nativas de América, y de ellas 32 correspondían a plantas chilenas y de estas, 27 eran plantas autóctonas" (Jiménez et al., 2016, p. 41).

Un nuevo texto fundacional de la farmacéutica nacional vio la luz en 1905 cuando Francisco Puga y Juan Bautista Miranda publicaron Farmacopea chilena y señalaron al respecto que esta debía ser el "Código Legal que debe tener todo país civilizado para regularizar el ejercicio de las dos profesiones médicas y para uniformar la acción y dosis terapéutica de los medicamentos" (1905, p. 9). A su vez, concibe esta nueva publicación la idea de que el medicamento tenga en sí la comprobación de sus efectos en el cuerpo y las enfermedades.

Ahora bien, una de las consecuencias de este esfuerzo por consolidar una farmacopea nacional con estándares internacionales fue que las prácticas curativas como el uso de yerbas autóctonas comenzaron una especie de retirada constante del uso doméstico, se les reemplazó por medicamentos creados en Chile o importados que se valieron tanto del voz a voz como de la publicidad en revistas que se analiza más adelante.

Sin embargo, el posicionamiento del campo farmacéutico para la vida cotidiana no solo se valió de la publicidad, sino también de un discurso de la salud (Foucault, 1996; 2002) que ya imperaba desde el nacimiento de la República. Por ejemplo, Guillermo Blest publicó en 1828 Ensayo sobre las causas más comunes y activas de las enfermedades que se padecen en Santiago de Chile, con indicación de los mejores medios para evitar la destructora influencia, el cual describe la dramática situación de salud y asistencia médica nacional en cuyo centro se encuentra el problema de la falta de un estándar de calidad internacional en la farmacopea nacional. A su vez, las incontables epidemias que azotaron a la población en este periodo (Cruz-Coke, 1995), sumada a la alta mortandad, hicieron de la medicina y farmacología una necesidad gubernamental.

En 1833 se instauró la carrera de Medicina y de Farmacia. Joaquín Tocornal, Ministro del Interior del Presidente Prieto, dictó el 19 de marzo de 1833 un decreto supremo por el cual el gobierno abría ambos cursos en el Instituto Nacional a cargo de José Vicente Bustillos. Con ello se comenzó a gestar la legitimación de un campo y una tecnología de la salud cotidiana que posteriormente generó el auge de las boticas 
a comienzos del siglo XX. En 1907 se contabilizaban más de cien boticas registradas en Santiago (Correa, 2016, p. 88), duplicaban así en número a las que había en algunas ciudades europeas, si se tiene en cuenta la proporción con respecto a cantidad de habitantes. Correa manifiesta incluso que la prensa de aquellos años hablaba de una población "emboticada desde su nacimiento" (Correa, 2016, p. 89). Por ello, muchos medicamentos se transformaron "en productos masivos que traspasaban los espacios que legalmente se definían como médicos o farmacéuticos" (Correa, 2016, p. 89) y se les utilizaba para todo tipo de síntoma.

Este problema de la proliferación de boticas y la necesidad del Estado de comenzar un proceso de profesionalización de estas, trajo consigo la posibilidad de mejorar también al medicamento como tecnología. Se obligó a las boticas a usar libros más especializados, organizarlos de acuerdo con sus tipos y enfermedades, además de contar con instrumentos de medición. En este sentido, se crearon las Comisiones Visitadoras de Boticas que paulatinamente fiscalizaron, por un lado, el quehacer farmacéutico y, por el otro, anularon al Protomedicato.

En las boticas se vendían principalmente, drogas simples, preparados en algunos casos de elaboración propia o terceros, específicos nacionales o extranjeros. Así, de una terapéutica ordinaria se pasó gradualmente a preparaciones más complejas que se acompañaron de un nuevo concepto: la dosimetría. Esta apelaba "a medicamentos precisos en sus dosis, hecho de gránulos solubles fáciles de tragar" (Correa, 2016, p. 93).

Por último, cabe destacar que si bien la Sociedad de Farmacia se fundó en Chile en 1854, presidida por José Vicente Bustillos, se podría señalar que en realidad su fundación comenzó hacia 1930 y en adelante, pues en 1945 se creó la primera Facultad de Química y Farmacia en la Universidad de Chile, aunque existen antecedentes que datan de 1891 que no prosperaron (Guzmán, 2003). Ello advierte que la farmacéutica tardó años en legitimarse como campo, sin embargo, se podría decir que el medicamento en tanto tecnología ya gozaba de reconocimiento poblacional, según lo mencionado con anterioridad.

\section{La revolución tecnológica del hogar y su impacto en las emociones}

Illouz (2007) plantea que las distintas transformaciones del capitalismo a nivel global, cuyo centro siempre se pensó en términos de subjetividades y flujo de capitales, tiene un ancla en lo que ella denomina una cultura emocional. Esto quiere decir que a cada razón económica de la época deviene de forma conjunta un discurso emociona,l el cual constituye un elemento esencial para el comportamiento económico individual o general.

De cierta forma, Illouz señala que el capitalismo emocional hizo que las emociones se vincularan de manera más estrecha con la acción instrumental. Cada decisión tiene un fundamento emocional (Maturana, 2001) y no solo obedece a una razón o estrategia 
anclada en un cálculo costo-beneficio. Por ejemplo, Hochschild (1997) manifiesta que en el trabajo elaborado por mujeres fuera y dentro del hogar, las emociones juegan un papel fundamental para que la acción permanezca en el tiempo. En su investigación en empresas estadounidenses, demuestra que cuando las mujeres argumentan que salen a trabajar para poder ayudar económicamente a sus familias, portan fotos de sus hijos en sus carteras a modo de recordatorio emocional por el esfuerzo diario. Esfuerzo, a su vez, se traduce en sentimientos de culpa por salir de casa. No obstante, también señalaban estas mujeres que el trabajo se había transformado en un espacio en el que eran reconocidas y encontraban emociones que habían desaparecido de sus hogares. Por ello el trabajo del hogar, o segundo turno como le denomina Hochschild (1989), si no fuera también por un ancla emocional, no tendría razones suficientes para llevarse a cabo dada tanta energía implicada en dicha actividad.

Por ello, Hochschild habla del advenimiento de un "trabajo emocional" (2008) cuyo fin es proporcionar el sentido necesario al trabajo, a la razón instrumental, para poder llevar a cabo distintas actividades. Tanto el trabajo en la empresa como el trabajo del hogar requieren una elaboración de un trabajo emocional que argumente la actividad. Allí, el trabajo de cuidado es quizás uno de los mejores ejemplos para comprender esta lógica, pues en este las emociones son más efectivas, en tanto que existe un trabajo sin remuneración bajo el argumento histórico-político de un amor instintivo y natural.

Ahora bien, cabe preguntar ¿cuál es la relación de la lógica emocional del capitalismo con el problema de la domesticación de los medicamentos en Chile a comienzos del siglo XX? Carrasco, Borderías y Torns (2011) señalan que el trabajo doméstico para su producción necesitó del mantenimiento físico de la familia y que para ello ha habido una gran circulación de mercancías dedicadas a la alimentación, la higiene o la salud de esta. Sin embargo, los autores pasan por alto la producción y el consumo masivo de fármacos durante todo el siglo XX, más bien destacan los alimentos infantiles, productos para el baño o cocina como tecnologías elaboradas para cuidar.

Sucede algo similar para Schwartz (2011) quien señala que durante el siglo pasado en Estados Unidos (aunque también en otras partes del mundo) hubo una revolución industrial que impactó en el hogar, producto de una transformación tecnológica de los aparatos domésticos. En la medida que ello ocurría surgieron ideologías que reforzaron pautas de conducta hacia la familia y la mujer en estrecha relación con la labor de cuidado. Lavadoras eléctricas, planchas o sistemas de agua caliente, tostadoras y hornos, entre otros, no solo acarrearon símbolos de estatus social, sino que también fueron una forma de liberar cierto trabajo en el hogar.

En la medida que esta revolución tomó forma, el número de personas por familia entró en declive, por lo que el trabajo de cuidado cobró importancia (y aquí las magazín 
fueron importantes en su configuración), pues hubo una presión implícita sobre la mujer bajo la siguiente ecuación: ahora que hay más tiempo gracias a las nuevas tecnologías, pueden cuidar mucho mejor a sus hijos. Es así que el cuidado como trabajo se fue especializando cada vez más y su ancla emocional se hizo cada vez más importante.

Ante tal presión, la mujer -sobre todo de una clase media-alta-comenzó a adquirir conocimientos expertos para que cuidar le fuera reconocido. De solo alimentar pasó a saber preparar fórmulas para que los bebes crezcan más fuertes. Supo esterilizar biberones para evitar infecciones y comenzó a obtener conocimientos de salud para poder hablar con médicos sobre sus hijos. Schwartzs (2011) señala que por ello el trabajo del hogar deviene cada vez más en una experiencia emocional, su ancla emotiva resultó ser una pieza fundamental para justificar la acumulación de conocimiento y dedicación temporal. Deriva de ello que cualquier problema que presenten los hijos, ahora se consideran el resultado de un descuido de la mujer, por ende, la culpa emerge como agravio personal y crítica social.

Así, bajo este entramado emergente entre tecnologías, cuidado, emociones y el discurso científico de la época en torno a la salud de la familia, emergió el magazín de comienzo de siglo que adoptó un rol importante en esta área.

Y, como veremos más adelante, con la emergencia de medicamentos de uso cotidiano la mujer ya no tenía excusas para sentirse cansada o irritada, pues ahora existía solución farmacológica para ello. La exigencia de estar alegre como madreesposa se hizo tan factible como la de propiciar una buena salud a los propios hijos. Por ello, cuando los medicamentos se introdujeron en el mundo del hogar y ofrecieron la posibilidad efectiva de la salud como imperativo, este tipo de tecnología superó su condición arraigada en la salud y se sumergió en un devenir de tecnología sensorial. Por ello, el amor a la familia y su cuidado supuso entonces como contra-fondo la medicalización imperiosa de la mujer que, a su vez, como expresión de su amor para con los suyos, sostuvo una medicalización del hogar.

Si, tal como advierte Schwartz (2011), la revolución industrial asociada al trabajo doméstico acarreó "cambios estructurales acusados en la fuerza de trabajo que aumentaron la carga de trabajo y ampliaron la descripción de las tareas para las trabajadoras" (p. 106), entonces su salud se transformó en ancla fundamental para el mantenimiento de la familia y del ritmo de la productividad del hogar.

Por ello, la conexión entre emociones y capitalismo como plantea Illouz (2007) cobra realidad en un espacio como el hogar (aunque no es el único) y es en la revista Familia en que se puede apreciar cómo se fue gestando este problema moderno. Para que los fármacos a comienzos del siglo Xx en Chile (y probablemente en otras partes del mundo) fueran entrando e involucrando en la vida cotidiana, la farmacéutica apuntó primero a la medicalización de la mujer como estrategia, para luego pasar a la medicalización de 
la familia a través de ella. Todo ello, sin embargo, no podría haber transcurrido sino fuera por el ancla emocional en la publicidad, como se verá a continuación.

\section{Emociones y publicidad: medicalizar a la mujer, medicalizar a la familia}

Hacia finales de 1910 y prácticamente durante todo 1911, comenzaron a aparecer anuncios publicitarios de boticas o farmacias en la prensa y revistas que advertían quizás una de las más grandes y silenciosas revoluciones del siglo XX: la producción de medicamentos (Meyer, 1986) para la vida cotidiana y el hogar.

No fue solo la medicalización del cuerpo-máquina (Fuster, 2014) lo que surgió con esta revolución, ni tampoco fue la lucha contra las enfermedades y la instauración de un cuerpo inmunizado, más bien fue un proceso paulatino de domesticación de una lógica y tecnología, de una razón médica que, como señala Fainzang (2013), implica hoy una "medicalización de sí". El horizonte normativo de esta idea es que la persona se transforma en médico de sí mismo y su existencia implica acudir al medicamento en busca de una solución eficaz para muchos de los problemas cotidianos.

Esta revolución silenciosa del campo farmacéutico para poder entrar al hogar necesitó, no obstante, ir paso a paso y para ello, recurrió a la arquitectura de la familia moderna: la mujer y la infancia, respectivamente. Por ejemplo, en 1910 aparecieron varios tipos de anuncios publicitarios en Familia en los que solo aparece el nombre de la botica y de un remedio que anunciaban que había llegado a sus bodegas, era el Oxijeno 3 de la botica Santo Domingo de la ciudad de Santiago. Situación similar ocurrió en la prensa, por ejemplo, en el periódico Novo doba (Biblioteca Nacional Digital de Chile, s. f.) de Punta Arenas de enero de 1911 se promocionó un reconstituyente del sistema nervioso llamado Neurosine Prunier de la botica Paris y Farmacias.

Sin embargo, luego comenzaron a utilizar cuerpos, personas y emociones asociadas a estas en las que la mujer, como se ya se adelantó, fue fundamental para la legitimación del campo farmacéutico. Ya para finales de 1910 un de los anuncios (ver figura 1) en la revista Familia exclamaba: "iCuidado, señora! Ud. empieza a engordar, y engordar es envejecer". Se promocionaba allí el fármaco Thyroydine Bouty del laboratorio francés Rue de Châteandun y en la imagen aparece una mujer con un ángel (con forma de niño o querubín) que está ajustando el vestido en la parte de la cintura. Surgen así dos ideas centrales con este primer anunciado: por un lado, el medicamento emerge como solución milagrosa para el cuerpo y por el otro, que engordar conlleva el peligro de envejecer, lo cual impulsa la idea de juventud como alegoría de salud y esbeltez.

Ver en la revista Familia n. ${ }^{\circ} 2$ de 1910. Se puede acceder a todos los números de Familia aquí referenciados en el Memoria chilena. Biblioteca Nacional de Chile (s. f.) en el siguiente enlace: http://www.memoriachilena.cl/602/w3-article-3415. html\#documentos 


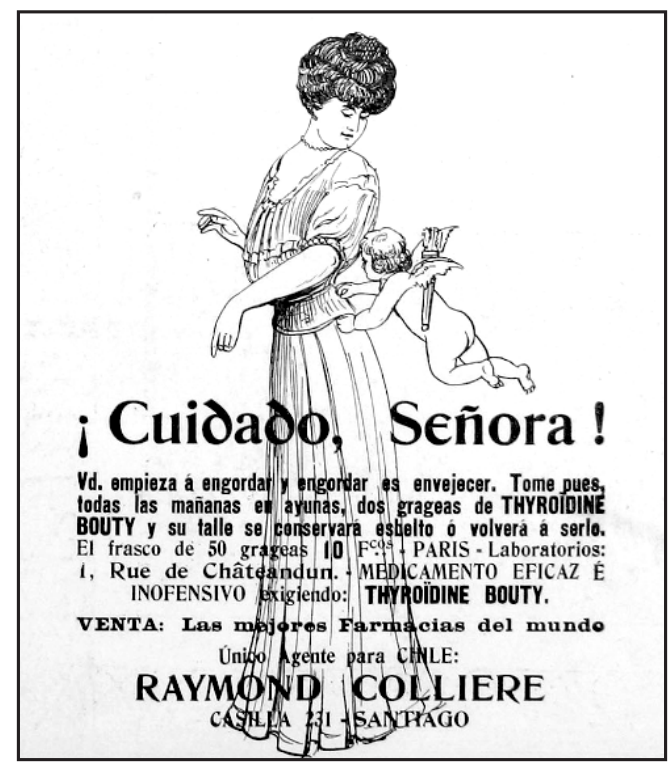

Figura 1. Publicidad en la revista Familia n. ${ }^{\circ} 14$ de 1910

Fuente: Fotografía Familia Santiago (Chile. 1910), Patrimonio Cultural común. Santiago de Chile, Chile.

Un segundo anuncio (ver figura 2) aparece en 1911 con una mujer sentada en un sillón cuya posición advierte malestar, sostiene su cabeza sobre su mano en señal de estar afligida. Se anuncia: "si esta señorita conociera los magníficos y seguros efectos de las auténticas Tabletas Bayer de Aspirina, no sufriría más de los insoportables dolores de cabeza". La asociación que se hace en esta publicidad es que las mujeres sufren dolores de cabeza cuyo impacto parece insufrible.

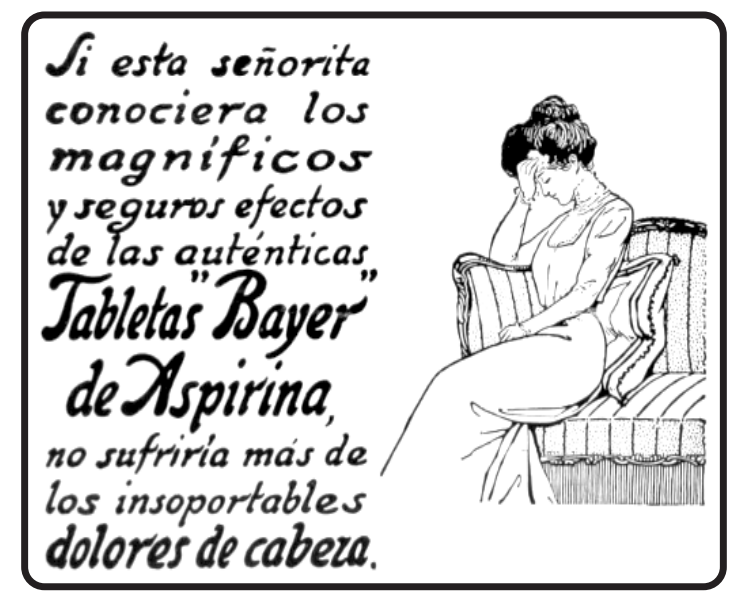

Figura 2. Publicidad en Familia n. 20 de 1911

Fuente: Fotografía de Jaime Montgomery (Chile, 1911), Patrimonio cultural común. Santiago de Chile, Chile. 
Dicha asociación mujer-dolor de cabeza performa hasta el día de hoy a la mujer. A los hombres prácticamente no se les asocia dicho malestar, que hoy en día tiene múltiples interlocutores o "soluciones" como Tapsin (Millones, 2018) u otros medicamentos. Con esta asociación se comenzó a construir una idea referente al comportamiento de la mujer, que se convierte en objeto de crítica social permanente: su amargura, inhabilidad, irritabilidad o lo que tenga como raíz el dolor de cabeza como principio, ahora tiene al fármaco como solución.

Así, muchos medicamentos que fueron apareciendo en Familia durante años insistieron en estas dos direcciones recién enunciadas: por un lado, la estética de la mujer (incluso se anuncian medicamentos para el aumento de senos como las Pilules Orientales en el n. ${ }^{\circ} 16$ de 1911 de la revista, disponible en Memoria chilena. Biblioteca Nacional de Chile, s. f.) y por el otro, en una normativa idealizada del género femenino que se traducía en cremas para mantener la piel joven, entre otras. Sin embargo, todos estos anuncios no evocaban emoción alguna de forma explícita, solo se hallaban en los bordes de las mismas y señalaban cierta frustración, cierto cuidado, cierta preocupación o cierto malestar, como en el caso de las aspirinas.

Para 1928 la farmacéutica Bayer ya presentaba cambios sustanciales en la forma de publicitar sus nuevos fármacos. Comenzó a aparecer una lógica emocional. Bayer anunciaba su fármaco Cafiaspirina y para ello evocaba, ya no un malestar como en el caso anterior de la Aspirina, sino que presentaba a una mujer sonriente y feliz (ver figura 3).

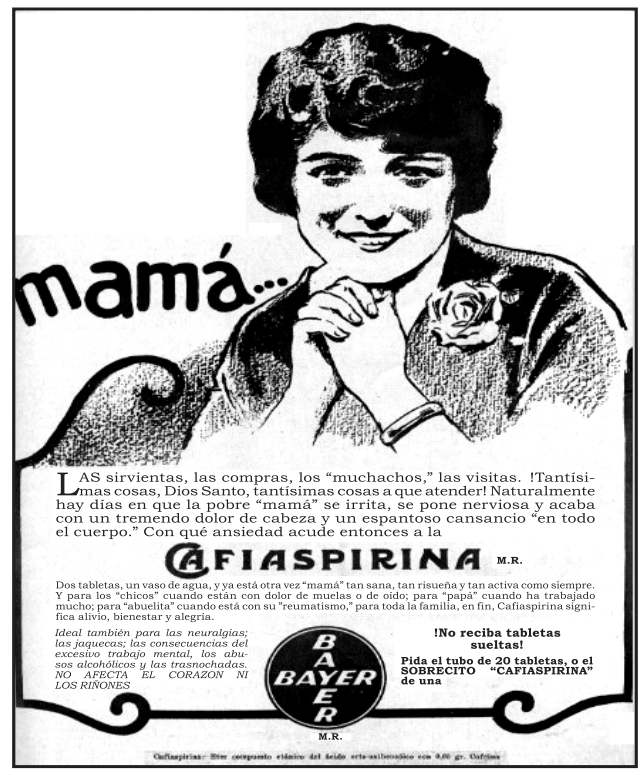

Figura 3. Publicidad en Familia n. ${ }^{\circ} 219$ de 1928

Fuente: Fotografía de José Marcos V. (Chile, 1928), Patrimonio cultural común. Santiago de Chile, Chile. 
La leyenda que le secunda a la imagen señala todas las cosas de las cuales debería estar pendiente una mujer-esposa-madre en su vida cotidiana: sirvientas, compras, hijos, visitas. Por todo eso y de manera tácita, el anuncio manifiesta que es natural que la madre padezca de irritabilidad, nerviosismo, cansancio y dolores de cabeza. La recomendación de Cafiaspirina supone así reponer una felicidad que parece esquiva. Se asociaron entonces, por un lado, tareas del hogar con malestar, por el otro, medicamento con alivio.

El medicamento en este caso ya no remedia exclusivamente un problema de salud, ya no se señalan aspectos estrictamente en este ámbito, sino que más bien lo que otorga el medicamento es una emoción deseada: ser una mujer-madre-esposa feliz. Creemos que en este periodo comenzó a gestarse una medicalización emocional que en algunos casos supera a la salud como motivo principal de consumo farmacológico, supedita la salud a las emociones, tanto post-consumo como previo-consumo. Por lo que, en este sentido, establecemos que en este periodo el medicamento inició una especie de carrera simbólica que prometía estados emocionales como principios consecuentes de la salud farmacológica.

Estos tipos de anuncios continuaron periódicamente durante muchos años y en todos los números de la revista Familia. Niños enfermos que se mejoraban por algún medicamento; niños fuertes gracias a algún fármaco o solución alimenticia; el paso de madres preocupadas y cansadas a madres bellas y alegres gracias a la acción de algún fármaco..., así pues, la presencia farmacológica en la familia tuvo una evolución en la que pasó del anuncio de la botica y algún medicamento en particular -que solo apelaba al problema de salud-a anuncios en los que las emociones se convirtieron en el centro y deseo proyectado. Fue de esta manera que la salud se empezó a asociar con las emociones y en ese paso, la mujer fue el centro de entrada de los medicamentos y la razón médico-farmacológica del hogar, y de gran parte de la vida cotidiana.

Al mismo tiempo que ocurría este movimiento, fue surgiendo de forma paralela otra vertiente que también involucró a la mujer. Ella, a su vez, se fue transformado en el nexo, en el puente, para la medicalización de toda la familia. A través de ella y del cuidado de la familia que recae sobre ella a manera de trabajo naturalizado, muchos medicamentos y productos -bajo una lógica médico-farmacéutica- se introdujeron en el hogar. Un ejemplo de ello es que a finales de 1912 apareció por primera vez en Familia una publicidad relacionada a la tos infantil (ver figura 4). El jarabe se llamaba Guayacose y también pertenecía a la farmacéutica alemana Bayer.

Advertían que el medicamento resultaba eficaz para combatir la bronquitis, la laringitis aguda e incluso la tuberculosis, mostraban con ello argumentos relacionados con la salud. En el anuncio se pueden identificar dos personas, una madre y un niño. La madre es quien tiene el jarabe en su poder y el niño se muestra pronto a recibirlo. Allí 
la mujer emerge como puente o nexo para la medicalización de la infancia. Obviamente no se trata solo de medicar al niño, sino de construir el imaginario de la buena madre cuyo ejercicio implica tener medicamentos a la mano para construir al niño sano y fuerte. Un niño sano es el resultado de una madre preparada. Una madre preparada, preocupada, debe tener una pequeña farmacia en su hogar.

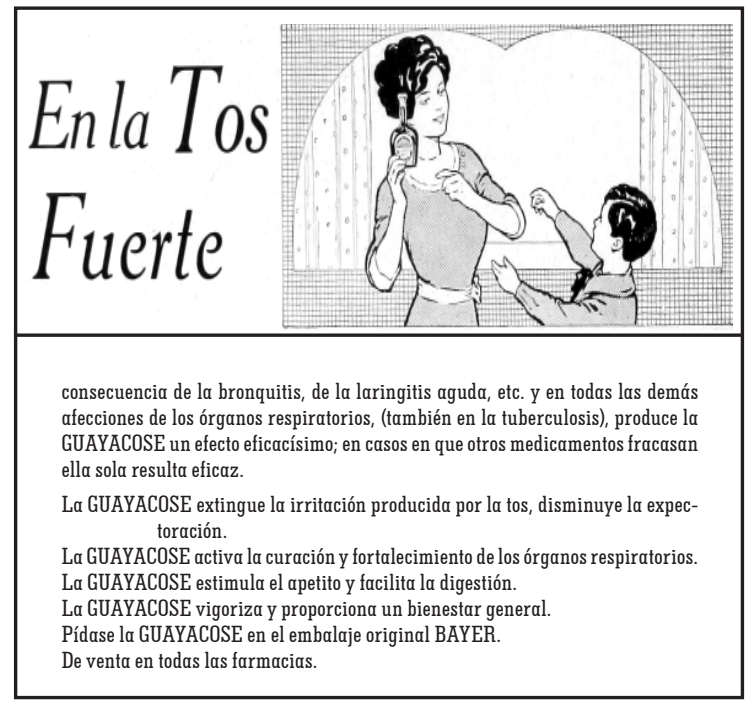

Figura 4. Publicidad en Familia n. 29 de 1912

Fuente: Fotografía publicitaria de Bayer (Chile, 1912), Patrimonio Cultural Común. Santiago de Chile, Chile.

A través de dicho puente, la infancia se vuelve objeto de medicalización y el ideal de niños fuertes se convierte en el argumento que se reiteró en incontables ocasiones para la promoción de distintos suplementos alimenticios. A modo de ejemplo, en 1935 apareció el anuncio de Cocoa Peptonizada Raff (en el n. ${ }^{\circ} 1$ de 1935 de la revista Familia, disponible en Memoria chilena. Biblioteca Nacional de Chile, s. f.). Primero se importó y luego en la década de 1930 la fabricó en Chile la empresa Comercial Guillermo Salinas y Cía. Es un producto que -en conjunto con otros-instauró la idea de una alimentación complementaria para que los menores crecieran más sanos. En 1937 se anunció Nutrinol (en el n. ${ }^{\circ} 111$ de 1937 de la revista Familia, disponible en Memoria chilena. Biblioteca Nacional de Chile, s. f.), se apeló a que el anhelo de la madre era el desarrollo robusto y sano de sus hijos. Dos advertencias surgieron a raíz de este anuncio: primero, la recomendación alimenticia se hacía desde un saber médico y este se presentó como garantía eficaz de sus efectos, ya que en el anuncio se señaló que el suplemento alimenticio era el "más recomendado por los médicos" para mejorar el crecimiento. Segundo, empezó a germinar de forma incipiente aún una idea de cuerpo como máquina (Fuster, 2013) que luego dió paso al abordaje del cuerpo como proyecto (Sibrian, 2016). 


\section{Emociones y capitalismo: una estrategia para la medicalización}

Correa (2016) señaló que a comienzos del siglo XX en muchos periódicos de la época se hablaba de Santiago de Chile como una sociedad emboticada debido a la gran cantidad de boticas que existían y que se estaban instalando. De cierta forma, es así como el paso de una sociedad emboticada a una sociedad farmacéutica no sorprendió, según este antecedente. El medicamento -sea elaborado o distribuido por estos espacios- tiene una importancia tal en la sociedad que aún no se le piensa en toda su complejidad.

Durante todo el siglo, la farmacología se convirtió así en un campo de gran potencial, no solo comercial sino que también con una gran capacidad para influir en la constitución corporal y subjetiva de nuestra cotidianidad, de nuestras relaciones e incluso nuestras expectativas. Solo piénsese en cómo la píldora anticonceptiva ha modificado la historia de la familia y de la vida cotidiana de las mujeres como para advertir la potencia de este tipo de tecnologías en el cuerpo, emociones y relaciones sociales.

Hoy los datos muestran que esta revolución no hace sino reafirmar esta tendencia. Para el 2018 en Chile se vendieron 1,8 millones de dosis farmacológicas diarias según el Instituto de Salud Pública (Chávez, 2018). Vivimos bajo ciertas formas que han hecho de la farmacia y el fármaco un espacio y tecnología necesarios para las proyecciones de nuestro día a día. Si, como dice Rose, nos hemos constituido en individuos somáticos (Rose, 2012) y hemos establecido una forma de vivir analgésica (Millones, 2018), la medicalización entonces no se centra solo en el medicamento y el dolor físico, también se ancla en las emociones. La medicalización de las emociones se muestra así como una estrategia crucial para que los medicamentos se puedan configurar como tecnologías del hogar que incluso sobrevivirán a muchas otras, puesto que su renovación implica siempre la salud como virtud.

Por ello, en el momento en el que las boticas comenzaron a introducir medicamentos para la vida cotidiana e incluyeron en su publicidad elementos emocionales como es el caso de la publicidad en Familia, en la que apuntan a la mujer-madre, se podría advertir que se comenzaron a gestar al menos dos efectos: 1) la exigencia de una mujer-madre reducida a ciertas emociones positivas como la alegría y 2) la posibilidad de crítica social por la forma en cómo se cuida de la familia, especialmente de la infancia. Esto es, aparece una obligatoriedad de que la mujer-madre esté alegre -ya existen fármacos para la irritabilidad y el cansancio-y segundo, se entregan fórmulas para que los hijos crezcan más sanos, por ende, existe la posibilidad de crítica a quien no siga dicha receta.

Illouz (2007) señala que en las emociones existe un proceso de racionalización cuyos elementos serían: 1) cálculo de los medios; 2) se utilizan los medios más efectivos; 3) se tiene un principio de valor general que constituya una guía para la vida y 4) se 
unifican todos estos elementos en una forma metódica y racional como forma subjetiva de explicación y resolución de problemas.

Al traducir la lógica planteada Illouz a la problemática referente a los medicamentos y emociones que hemos planteado, se podría manifestar que, en primer lugar, el cálculo que haría la mujer para medicalizarse a ella misma y también a su familia podría estar relacionado a cierto cálculo emocional promovido por la crítica y culpa que pueden emerger si no hace lo que se debería hacer para el cuidado de sus menores. Es decir, las tareas del hogar no son impedimentos para el cuidado, puesto que estar irritable o cansada debido a dicho trabajo ya tiene solución farmacológica. Segundo, cuando se habla del uso de medios efectivos, sin duda el medicamento se posiciona -al incluir o soportarse en el discurso médico- con cierta ventaja frente a otras opciones como el conocimiento indígena medicinal, por la idea de garantía moderna. Tercero, si existe un principio de valor general moderno, la salud se constituye quizás como la principal guía para la vida, en tanto que virtud y promotor de los avances de la sociedad. Y finalmente, confluyen todos estos elementos en la forma de medicalización en sí como expresión de una subjetividad moderna (Fainzang, 2013), de explicación y resolución de problemas de la vida cotidiana.

De esta manera, Familia adoptó la forma de consejo profesional, de receta emocional, no solo para sanar enfermedades o aliviar dolores, sino para transformar modos de vivir asociados o respaldados por el discurso médico en el cual los medicamentos se erigieron como prueba efectiva de ello. Todo, sin duda alguna, bajo un apremio cultural de cuidado emocional de la familia que la revolución industrial del hogar le endilgó a las mujeres, como señala Schwartz (2011), amarrado a una crítica al acecho, si es que no se llevan a cabo ciertas acciones como las hasta ahora ya refrendadas.

En este sentido y para ir finalizando, creemos que medicalizar a la mujer en tanto que mujer y luego como madre fue una clara estrategia del campo farmacéutico para legitimarse y así poder entrar al hogar. Domesticar al fármaco tuvo como necesidad la gestación de una medicalización emocional. El capitalismo moderno, en este sentido, necesitó de un ancla emocional para circular sus tecnologías. Sin embargo, ello no podría haber sido posible sino es por la misma carga emocional de la crítica y culpa de y hacia la mujer por no cumplir su rol coaccionado: no hay excusas para el dolor de cabeza, para estar cansada o irritable; ahora la solución farmacológica exhibe su efectividad como promesa.

Por último, la felicidad, la seguridad o la plenitud, ser quien se desea ser, puede lograrse a través de medicamentos o quizás al menos acercarse a una administración de dichas emociones a través de una farmacología del hogar. Y en Familia, creemos, se dio nido al espacio como testimonio de este avanzar en la administración emocional y tecnológica de la vida actual. 


\section{Conclusiones}

La gran mayoría de los anuncios publicitarios farmacológicos que aparecen en toda la historia de la revista Familia se enfocaron en la mujer. En algunas ocasiones aparecieron junto a sus hijos, maridos o solas. También hubo algunas publicidades en las que solo aparecía un hombre o el fármaco. El asunto no es en sí probabilístico, aunque por ser una revista pensada para mujeres, muchas de sus publicidades apuntan a ellas, sean o no farmacológicas.

Ahora bien, el naciente uso de emociones como estrategia del capitalismo de comienzo del siglo xx en Chile ${ }^{4}$, fue dando pistas de que los fármacos poco a poco irían sumándose a una carrera en la que todo parecería tener una solución farmacológica.

El hecho de que los anuncios publicitarios en Familia utilizaran un ancla emocional como estrategia de legitimación de un campo advierte que estas eran de tipo social, coyunturales y relacionales (Bericat, 2000). Esto implica la posibilidad de repensar muchas discusiones clásicas en relación a la historia de la mujer y la familia, el trabajo y muchos otros escenarios a partir del cómo se han construido, gestado y administrado las emociones involucradas.

Desde esta perspectiva, la medicalización de la familia y de la mujer particularmente, incluye un componente emocional que va más allá de la salud en sí y que involucra la configuración de roles en cada sociedad. Sin duda alguna, lo planteado aquí no explica el consumo de fármacos en la vida cotidiana en su totalidad. Existen razones más profundas en este fenómeno, físicas, antropológicas, sociológicas, económicas, etc. No obstante, la relación entre emociones y capitalismo, emociones y tecnología, debe empezar a ser revisada, creemos que lo que sucede con los fármacos quizás ha sucedido también con otras tecnologías, aunque a diferencia de otras, el fármaco goza de una legitimidad ontológica que podría incidir en su consumo sin mucha regulación.

\section{Referencias}

Abraham, J. (2010). Pharmaceuticalization of society in context: theoretical, empirical and health dimension. Sociology, 44(4), 603-624. https://doi.org/10.1177/0038038510369368

Alliende, M. (1987). Un caso particular: historia de la farmoquímica del Pacífico S. A. (1834-1987). Edición de la Academia de Ciencias Farmacéuticas.

Anderson, B. (1991). Imagined communities. Reflections on the origin and spread of nationalism. Verso.

Bericat, E. (2000). La sociología de la emoción y la emoción en la sociología. Paper, 62, 145-176.

Es probable que esta dinámica haya sido más o menos similar en otros países de América Latina, Europa o Estados Unidos, debido a que muchos fármacos eran importados precisamente desde los últimos dos al continente sudamericano, medicamentos que apuntaban a solucionar distintos problemas de la vida cotidiana. 
Biblioteca Nacional Digital de Chile. (s. f.). Novo doba. http://www.bibliotecanacionaldigital.cl/ bnd/633/w3-article-158492.html

Bourdieu, P. (1996). Cosas dichas. Gedisa Editorial.

Carrasco, C., Borderías, C. y Torns, T. (2011). El trabajo de cuidados. Historia, teoría y políticas. Cataratas.

Chávez, M. (2018, septiembre). Chilenos compraron más de 1,8 millones de pastillas al día durante el primer trimestre. El Mercurio, Economía y Negocios. http://www.economiaynegocios. $\mathrm{cl} /$ noticias/noticias.asp?id $=506175 \#$

Conrad, P. (2007). The medicalization of society. On the transformation of human condition into treatable disorders. The John Hopkins University Press.

Correa, M. J. (2016). "Brulote disfrazado, no oblea medicinal". El avance de los específicos en el Chile urbano del Centenario. En Y. Carvajal y M. J. Correa (eds.), Historia de los medicamentos. Apropiaciones e invenciones en Chile, Argentina y Perú (pp. 85-108). Ocholibros.

Fainzang S. (2013). The Other Side of Medicalization: Self-Medicalization and Self-Medication. Culture, medicine and psychiatry, 37(3), 488-504. https://doi.org/10.1007/s1 1013-013-9330-2

Foucault, M. (1996). La vida de los hombres infames. Altamira.

Foucault, M. (2002). Defender la sociedad. FCE.

Fox, N., y Ward, K. (2008). Pharma in bethroom... and the kitchen... The pharmaceuticalization of daily life. Sociology of Health and Illness, 30(6), 856 868. https://doi.org/10.1111/j.14679566.2008.01114.x

Fuster, N. (2013). El cuerpo como máquina. La medicalización de la fuerza de trabajo en Chile. Ceibo Ediciones.

Fuster, N., y Moscoso-Flores, P. (2015). La hoja sanitaria. Archivo del policlínico obrero de la I. W. W. Chile 1924-1927. Ceibo Ediciones.

García Huidobro, C., y Escobar, P. (2012). Una historia de las revistas chilenas. Universidad Diego Portales.

Guzmán, E. (2003). Historia de una profesión. Colegio Químico Farmacéutico y Bioquímico de Chile A. G. 60 años 1942-2002. Editorial Trineo.

Hochschild, A. (1997). The time bind. When work become home and home become work. A Holt Paperback. Hochschild, A. (1998). The second shift. Avon.

Hochschild, A. (2008). La mercantilización de la vida íntima. Apuntes de la casa y el trabajo. Katz Editores. Illouz, E. (2007). Intimidades congeladas. Las emociones en el capitalismo. Katz Editores.

Jiménez, J. F., Alioto, S., Parada, M., Villar, L., Carreño, I., Muñoz, R. y Carvajal, Y. (2016). Herbolarias originarias y farmacologías modernas: presencia, apropiaciones y devoluciones en el caso de Chile. En Y. Carvajal y M. J. Correa (eds.). Historia de los medicamentos. Apropiaciones e invenciones en Chile, Argentina y Perú (pp. 15-52). Ocholibros. https://www.academia.edu/39981059/ Herbolarias_originarias_y_farmacolog\%C3\%ADas_modernas_presencias_apropiaciones_y_ devoluciones_en_el_caso_de_chile 
Maturana, H. (2001). Emociones y lenguaje en Educación y Política. Dolmen Ediciones.

Memoria chilena. Biblioteca Nacional de Chile (s. f.). Entre el hogar y las letras. Familia (19101928) (1935-1940). Biblioteca Nacional Digital de Chile. http://www.memoriachilena.gob.cl/602/ w3-article-3415.html\#documentos

Meyer, P. (1986). La revolución de los medicamentos. Mitos y realidades. Espasa.

Millones, M. (2018). Vidas medicalizadas: desde la mirada médica a una vida analgésica. Revista Latinoamericana de Estudios sobre Cuerpo, Emociones y Sociedad, 10(27), 10-20. https://dialnet. unirioja.es/descarga/articulo/6983992.pdf

Montero, C. (2013). Cincuenta años de prensa de mujeres en Chile, 1900-1950. En A. Stuven y J. Fermandois (eds.), Historia de las mujeres en Chile (pp. 319-353). Taurus.

Schwartz, R. (2011). La revolución industrial del hogar: tecnología doméstica y cambio social en el siglo XX. En C. Carrasco, C. Borderías y T. Torns (eds.), El trabajo de cuidados. Historia, teoría y políticas (97-121). Cataratas.

Orellana, T. (2016). Estereotipos femeninos de salud y belleza en Chile a través del género magazine: Revista Familia (1910 1928). En C. Arayay, C. Leyton, M. López, C. Palacios y M. Sánchez (eds.), República de la salud. Fundación y ruinas de un país sanitario. Chile siglos XIX y XX (pp. 226-256). Ocholibros Editores.

Ossandón, C. y Santa Cruz, E. (2005). El estallido de las formas. Chile en los albores de la cultura de masas. Lom.

Puga, F., y Miranda, J. (1905). Farmacopea chilena. Imprenta Barcelona.

Rose, N. (2012). Políticas de la vida: biomedicina, poder y subjetividad en el siglo XXI. Unipe, Editorial Universitaria.

Sánchez, M. (2016). Instrucciones para la vida. Normas prácticas, morales y políticas en un almanaque farmacéutico chileno, 1920-1930. En. Y. Carvajal y M. J. Correa (eds.), Historia de los medicamentos. Apropiaciones e invenciones en Chile, Argentina y Perú (pp. 152 156). Ocholibros.

Sibrian, N. (2016). De máquina a proyecto: el cuerpo en el nuevo espíritu del capitalismo. Reflexiones, 25(1), 143-155. https://doi.org/10.15517/RR.V95I1.27659

Tobar, T. (2015). Distribución de farmacias por región. Situación a diciembre 2014. Ministerio de Salud, Gobierno de Chile. https://www.minsal.cl/sites/default/files/files/2015_01_\%20 DISTRIBUCI\%C3\%93N\%20FARMACIAS\%20Situaci\%C3\%B3n\%20Diciembre\%202014\%20TTA. PDF 